\title{
Mechanisms of peritoneal dissemination in gastric cancer (Review)
}

\author{
FENG SUN, MIN FENG and WENXIAN GUAN \\ Department of General Surgery, Nanjing Drum Tower Hospital, The Affiliated Hospital of \\ Nanjing University Medical School, Nanjing, Jiangsu 210008, P.R. China \\ Received October 15, 2015; Accepted March 21, 2017
}

DOI: $10.3892 / \mathrm{ol} .2017 .7149$

\begin{abstract}
Peritoneal dissemination is the most frequent metastatic pattern of gastric cancer, but the mechanisms underlying peritoneal dissemination are yet to be elucidated. Paget's 'seed and soil' hypothesis is recognized as the fundamental theory of metastasis. The 'seeding' theory proposes that the formation of peritoneal dissemination is a multistep process, including detachment from the primary tumour, transmigration and attachment to the distant peritoneum, invasion into subperitoneal tissue and proliferation with blood vascular neogenesis. In the present review, the progress of each step is discussed. Milky spots, as a lymphatic apparatus, are indicative of lymphatic orifices on the surface of the peritoneum. These stomata are open gates for peritoneal-free cancer cells to migrate into the submesothelial space. Therefore, milky spots provide suitable 'soil' for cancer cells to implant. Other theories have also been proposed to clarify the peritoneal dissemination process, including the transvessel metastasis theory, which suggests that the peritoneal metastasis of gastric cancer develops via a vascular network mediated by hypoxia inducible factor- $1 \alpha$.
\end{abstract}

\section{Contents}

\author{
1. Introduction \\ 2. Detachment from the primary tumour \\ 3. Transmigration to the distant peritoneum \\ 4. Adhesion to the peritoneum \\ 5. Invasion into subperitoneal tissue \\ 6. Promotion of metastasis by $\mathrm{CAF}$ \\ 7. Milky spots \\ 8. Involvement of microRNA in peritoneal dissemination \\ 9. Transvessel metastasis theory \\ 10. Conclusion
}

Correspondence to: Dr Wenxian Guan, Department of General Surgery, Nanjing Drum Tower Hospital, The Affiliated Hospital of Nanjing University Medical School, 321 Zhongshan Road, Nanjing, Jiangsu 210008, P.R. China

E-mail: medguanwx@163.com

Key words: gastric cancer, peritoneal dissemination, epithelialmesenchymal transition, cancer-associated fibroblasts, milky spots

\section{Introduction}

There were an estimated $\sim 1$ million incident diagnoses of gastric cancer globally in 2012, half of which occurred in Eastern Asia, particularly in China (1). Although the incidence of gastric cancer has reduced in the past few decades, it remains the 5th most frequently diagnosed type of cancer and the 3rd leading cause of cancer-associated mortality globally (1). Peritoneal dissemination is the typical metastatic pattern in gastric cancer and data obtained from between 1969 and 1988 in Japan indicated that peritoneal recurrence accounted for $>40 \%$ of recurrence cases $(2,3)$. To date, there is no standard treatment for peritoneal metastasis. Therefore, investigating the underlying mechanisms of peritoneal dissemination is required for efficient treatment and to further improve the prognosis of patients. Paget's 'seed and soil' theory has been established as the fundamental theory of peritoneal dissemination in gastric cancer $(4,5)$. This hypothesis compares the viable cancer cells to 'seeds' and the suitable microenvironment of the metastatic sites to 'soil' (6). The current review will thoroughly discuss the underlying mechanisms of peritoneal dissemination in gastric cancer, based on the 'seed and soil' theory.

The 'seeding' theory demonstrates that several consecutive steps participate in the development of peritoneal dissemination, including detachment from the primary tumour, transmigration to the peritoneum, attachment to peritoneal mesothelial cells or lymphatic stomata, invasion through the basement membrane to subperitoneal tissue and proliferation with blood vascular neogenesis (Fig. 1) (7). Finally, the progress of these processes is discussed.

\section{Detachment from the primary tumour}

Detachment of cancer cells from the primary tumour is considered the 1st step of peritoneal dissemination. These detached cancer cells are designated peritoneal-free cancer cells and the positive rate of cytological examination is associated with the depth of tumour invasion and degree of differentiation (8). With the recent rapid development of molecular biology techniques, various novel tumour markers have been identified to be predictors of subclinical peritoneal dissemination in gastric cancer. Epithelial (E)-cadherin mediates the adherens junction between homophilic cells, inhibiting the ability of gastric cancer cells to detach from the primary tumour. S100 calcium binding protein A4 (S100A4) affects cancer cell motility via the alteration of cytoskeletal dynamics. Gastric cancer cells 


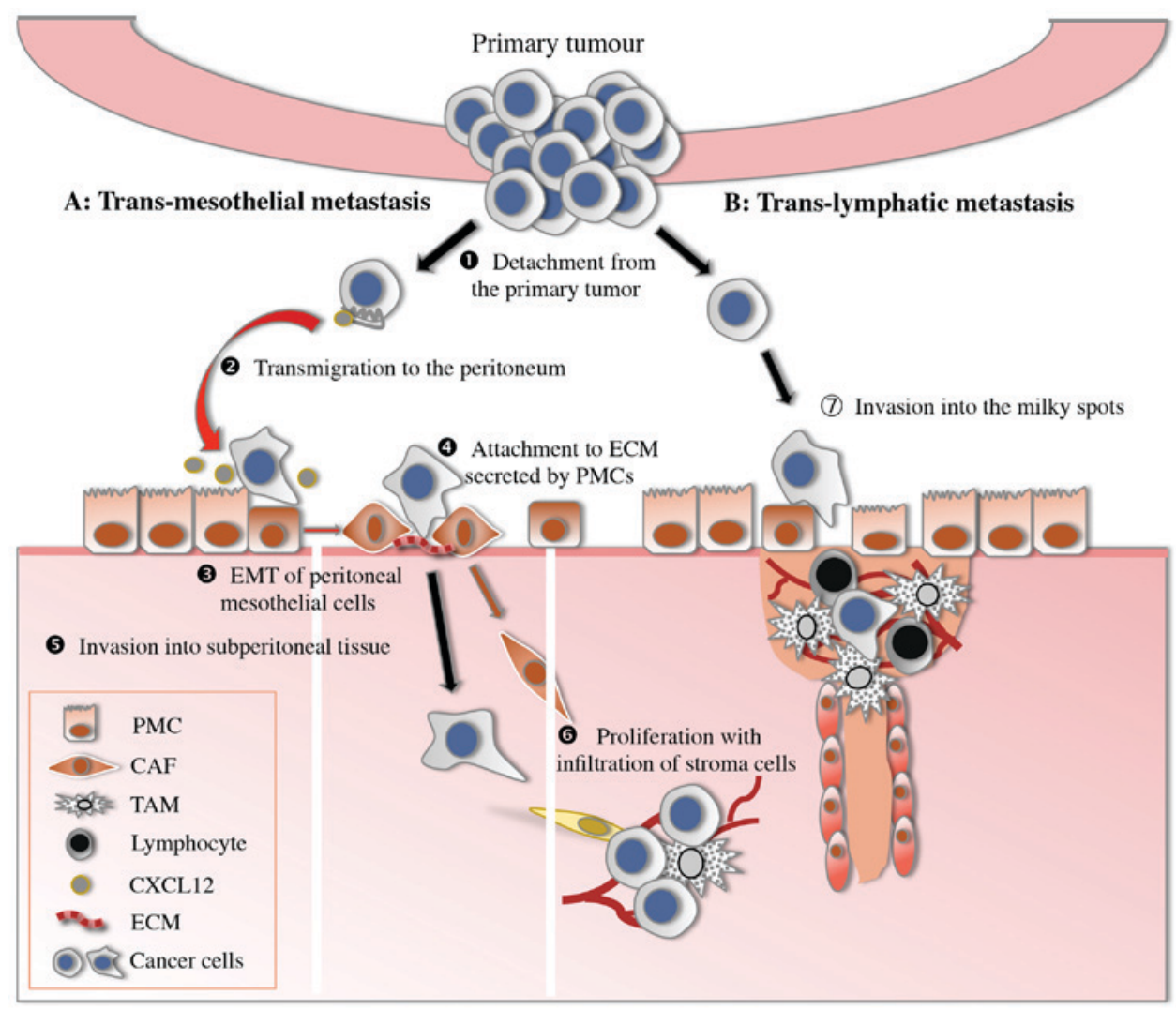

Figure 1. Peritoneal dissemination model of gastric cancer. (1) Detachment from the primary tumour (E-cadherin, S100A4). (2) Transmigration to the distant peritoneum (CXCL12/CXCR4). (3) EMT of peritoneal mesothelial cells (TGF- $\beta 1$, CD44). (4) Attachment of gastric cancer cells to the ECM factors secreted by PMCs (integrin). (5) Invasion into subperitoneal tissue (MMP, motility factors). (6) Proliferation with infiltration of stromal cells, particularly CAF (CXCL12/CXCR4). (7) Invasion into the milky spots through lymphatic orifices. EMT, epithelial-mesenchymal transition; ECM, extracellular matrix; PMC, peritoneal mesothelial cells; CAF, cancer-associated fibroblasts; TAM, tumour-associated macrophages; E-cadherin, epithelial-cadherin; S100A4, S100 calcium binding protein A4; CXCL12, C-X-C motif chemokine ligand 12; CXCR4, C-X-C motif chemokine receptor 4; TGF, transforming growth factor; CD, cluster of differentiation; MMP, metalloproteinases.

with downregulated epithelial (E)-cadherin and upregulated S100A4 expression have an increased probability of undergoing serosal involvement and peritoneal dissemination (9).

Primary tumours are the principal source of peritoneal-free cancer cells; two other sources are metastatic lymph nodes and surgical dissection $(10,11)$. For patients without serosal invasion, Marutsuka et al (10) demonstrated that lymph node dissection opens lymphatic channels, which spreads cancer cells into the abdominal cavity. A recent study performed cytology analysis of peritoneal wash fluid via reverse transcription-quantitative polymerase chain reaction (RT-PCR) prior to and following a gastrectomy, and demonstrated that, of 57 peritoneal wash samples with no carcinoembryonic antigen (CEA) or cytokeratin (CK) 20 prior to gastrectomy, 35 samples had CEA or CK20 positivity following the gastrectomy (11). These results confirmed that surgery may induce viable cancer cell efflux into the peritoneal cavity during a curative gastrectomy (11). Therefore, it is important for surgeons to adhere to surgical standards and perform the required actions to eliminate these viable cancer cells during surgery using modalities such as hyperthermic intraperitoneal chemotherapy.

\section{Transmigration to the distant peritoneum}

The next step of peritoneal dissemination is transmigration of peritoneal-free cancer cells to the distant peritoneum. The sites where cancer cells adhere are not randomly selected but are promoted by chemokines and corresponding receptors $(12,13)$. Chemokines and their receptors have an important role in promoting the migration of leucocytes (14). However, chemokine receptors have recently been the focus of attention as the expression of chemokines receptors on cancer cells, particularly C-X-C motif chemokine receptor 4 (CXCR4), is associated with cancer cell migration and metastasis (15). CXCR4 was first reported as a chemokine receptor that participates in the lung metastasis of breast cancer (16). An increasing number of studies have demonstrated that the CXC chemokine ligand 12 (CXCL12)/CXCR4 axis participates in the development of peritoneal dissemination in gastric cancer (17). It is hypothesized that CXCL12 is produced by peritoneal mesothelial cells and attracts gastric cancer cells expressing CXCR4 (17). The positive rate of CXCR4 expression is significantly higher in primary gastric cancer with peritoneal metastasis (67\%), compared with other types of distant metastasis (25\%) (15). Although $85 \%$ of patients who are positive for CXCR4 develop peritoneal metastasis, the proportion of patients who are negative for CXCR4 is only 48\% (15). In addition, high concentrations of CXCL12 are detected in malignant ascitic fluids (15). These results are concordant with the CXCL12/CXCR4 axis serving an important function in the peritoneal dissemination in gastric cancer; therefore, CXCR4 may be a novel target for treatment. 


\section{Adhesion to the peritoneum}

Adhesion of gastric cancer cells to the peritoneal lining is a crucial step in peritoneal dissemination. Formation of peritoneal dissemination from peritoneal-free gastric cancer cells is considered to occur through two distinct processes: Transmesothelial and translymphatic metastasis.

Transmesothelial metastasis originates from the attachment of peritoneal-free cancer cells to the mesothelium (18). Human peritoneal mesothelial cells (HPMCs) have a vital role in this process. Normal peritoneum is composed of a single layer of mesothelial cells closely connecting with each other, creating an anatomical barrier that prevents peritoneal-free gastric cancer cells from invading into submesothelial tissues (19). However, the morphology of HPMCs is altered by stimulation with a number of the growth factors secreted by cancer cells. The alteration in morphology from epithelial-like to mesenchymal-like is termed the epithelial-mesenchymal transition (EMT) (20). The EMT is characterized by the attenuation of E-cadherin expression and elevation of $\alpha$-smooth muscle actin (SMA) expression (20). HPMCs that have undergone EMT take on a spindle-like, fibroblastic-pattern morphology, which promotes a space between mesothelial cells and exposes the basement membrane to cancer cells (21). Transforming growth factor $\beta 1$ (TGF- $\beta 1$ ) serves an important role in the progress of EMT (22).

TGF- $\beta 1$ is part of the TGF- $\beta$ superfamily that controls the proliferation and differentiation of the majority of cells; it is a crucial promoter in several fibrotic diseases (23). TGF- $\beta 1$ has been established to promote the expression of extracellular matrix (ECM) factors, including collagen, through Smad3 and the conversion of renal tubular epithelial cells into fibroblasts in the kidney via Smad2 (24). Regarding gastric cancer, TGF- $\beta 1$ levels detected using an ELISA with peritoneal wash fluid samples are associated with peritoneal dissemination and depth of invasion (18). Furthermore, TGF- $\beta 1$ induces the EMT of HPMCs in vitro and in vivo (25). Subsequent previous studies have further demonstrated that TGF- $\beta 1$ increases the expression of collagen I in rat peritoneal mesothelial cells via the c-Jun $\mathrm{N}$-terminal kinase $(\mathrm{JNK}) / \mathrm{Smad} 3$ signalling pathway, and of collagen III and fibronectin of HPMCs via Smad2 $(26,27)$. Consequently, mesothelial cells stimulated by TGF- $\beta 1$ promote peritoneal fibrosis via upregulating the expression of ECM proteins, including collagen I, collagen III and fibronectin (18). Cancer cells may anchor to the ECM via adhesion molecules; therefore peritoneal fibrosis mediated by TGF- $\beta 1$ provides an effective 'soil' for dissemination.

Several regulatory factors modulate the function of TGF- $\beta 1$, including connective tissue growth factor (CTGF) (28), angiotensin II (Ang II) (29) and phytosulphokine (PSK) (30). CTGF is an important downstream effector of TGF- $\beta 1$. A previous study demonstrated that CTGF induces the EMT of HPMCs directly and that EMT that is mediated by TGF- $\beta 1$ is reversed when the cells are transfected with CTGH small interfering (si)RNA (28). These results are concordant with CTGF participating in the progress of EMT, mediated by TGF- $\beta 1$. Ang II is a member of the renin-angiotensin system (RAS) and the identification of local RAS in cancer tissues brings more attention to the function of Ang II in the development of cancer. Local RAS indicates that Ang II is converted directly from Ang I in tumour tissues in an angiotensin-converting enzyme
(ACE)-independent manner. In vitro studies have demonstrated that Ang II increases the expression levels of TGF- $\beta 1$ and is inhibited by candesartan, a type of angiotensin II type 1 block (ARB) (29). These results indicated that ARBs may be used to treat fibrosis in peritoneal dissemination (29). Protein-bound polysaccharide K (PSK), used as an anticancer immunologic adjuvant therapy, is considered to prolong the survival of patients with gastric cancer (31). PSK has been established to suppress the TGF signalling pathway (32). Shinbo et al (30) reported that PSK inhibits the EMT of HPMCs induced by TGF- $\beta 1$ in vitro, and the co-inoculum of gastric cancer cells and HPMCs significantly inhibits fibrosis in xenograft tumours. These results suggest that PSK may be used to control tumour fibrosis in gastric cancer.

Cluster of differentiation (CD)44, a type of adhesion molecule produced by gastric cancer cells, also contributes to the contraction of mesothelial cells by phosphorylating the cellular skeleton (33). All of these factors result in morphological alteration of HPMCs and peritoneal fibrosis, which provides suitable 'soil' for peritoneal-free cancer cells. Consequently, cancer cells migrate through a layer of mesothelial cells and further attach to the ECM expressed by HPMCs to expose the basement membrane mediated by integrin molecules (34). Integrins are membrane-bound proteins that mediate the direct contact between cells and the extracellular matrix (34). Integrins are composed of two distinct chains, the $\alpha$ and $\beta$ subunits (35). There are $18 \alpha$-integrin subunits in mammals. Amongst these subunits, $\alpha 1, \alpha 2, \alpha 3$ and $\beta 1$ have been reported to be closely associated with the peritoneal dissemination of gastric cancer $(36,37)$, particularly $\alpha 3 \beta 1$ integrin (34), which mediates gastric cancer cell adhesion to laminin. A recent study identified that CTGF effectively blocks adhesion by binding to $\alpha 3 \beta 1$ integrin, and the authors hypothesized that recombinant CTGF may have therapeutic potential (38).

\section{Invasion into subperitoneal tissue}

Degradation of the ECM and the basement membrane is beneficial for the invasion of cancer cells. Matrix metalloproteinases (MMPs) are able to degrade all types of ECM. MMP7, and MMP14 are synthesized primarily by cancer cells, whilst other MMPs are synthesized by cancer cells and stromal cells (39). The level of MMP7 in peritoneal lavage was detected using RT-PCR and the results demonstrated that MMP7 expression is associated with peritoneal metastasis, for which the sensitivity was $53.3 \%$ (40). In addition, the expression of MMP14, as detected using RT-PCR of peripheral blood and bone marrow samples, is associated with peritoneal dissemination in gastric cancer (41). MMPs are suppressed by tissue inhibitor metalloproteinases (TIMPs) (42). Miyagi et al (43) transfected the TIMP-1 gene into gastric cancer cells using adenoviral vectors and identified that the cell invasion ability was reduced, as analysed using an in vitro invasion assay, and the weight of the metastasis peritoneal nodes was also significantly reduced in in vivo studies, compared with those in the control group. Notably, when detecting TIMP-1 concentrations in tumour tissues using ELISA, high levels of TIMP-1 are associated with peritoneal dissemination and an unfavourable prognosis (44). Consequently, MMP7/MMP14 and TIMP-1 may be used as efficient markers to predict peritoneal dissemination. 
Gastric cancer cells require the ability to migrate in order to invade the gastric wall and subperitoneal tissue. Motility factors are expressed by cancer cells to promote cell invasion and migration. c-MET, also known as hepatocyte growth factor (HGF) receptor, is a membrane receptor that participates in the development of certain types of disease, including cancer, autism, and cardiac dysfunction (45). HGF is the only established ligand of c-MET (46). High expression levels of HGF and c-MET are associated with peritoneal dissemination and a reduced median survival time (47). Toiyama et al (47) demonstrated that HGF promotes invasiveness, the migration of gastric cancer cells and the inhibition of anoikis, which was inhibited by the kinase inhibitor SU11274.

Brain-derived neurotrophic factor (BDNF) is part of the neurotrophin family of growth factors and has been demonstrated to bind to $>2$ receptors, including tropomyosin-related kinase B (TrkB) and low affinity nerve growth factor receptor (48). BDNF expression levels in the invasive cells of tumours are significantly increased compared with those in the core cells and in the normal mucosa (49). In addition, co-expression of BDNF/TrkB is associated with the prognosis of gastric cancer (49). This study has also confirmed that BDNF promotes the proliferation, migration and invasion of gastric cancer cells that express TrkB in vitro, and that the Trk antagonist K252 significantly decreased the tumour size and the number of peritoneal dissemination sites, compared with the control group, in vivo (49).

Annexin A1 (AnxA1) is a member of the $\mathrm{Ca}^{2+}$-dependent phospholipid-binding protein family and was initially considered to be a glucocorticoid-regulated anti-inflammatory protein (50). Recently, AnxA1 has been of increasing relevance as it has diverse effects on cellular functions (51). Cheng et al (52) demonstrated that elevated expression levels of AnxA1 were associated with peritoneal dissemination. Results in this study also identified that AnxA1 activates the formyl peptide receptor signals, which further activate the mitogen-activated protein kinase 1 (ERK)/integrin $\beta$-1-binding protein 1 pathway to promote the migration and invasion of gastric cancer cells (52).

When gastric cancer cells invade into subperitoneal tissue, growth factors secreted by cancer cells and stromal cells, including vascular endothelial growth factor (VEGF)-A and VEGF-C, further promote cell proliferation and vascular neogenesis (53).

\section{Promotion of metastasis by CAF}

It is well established that tumour tissues contain cancer cells and cancer stroma (54). Interactions between the cancer cells and cancer stroma induce the processes of invasion and migration. The stroma is comprised of numerous types of mesenchymal cells and extracellular matrix. Cells of the cancer stroma include fibroblasts, immune cells, endothelial cells and other types of bone marrow-derived cells (55). Carcinoma-associated fibroblasts (CAFs), which consist of both fibroblasts and myofibroblasts, are frequently observed in the stroma of human carcinomas (56). Myofibroblasts, as the form of activated fibroblasts, are characterized by the overexpression of $\alpha$-smooth muscle actin ( $\alpha$-SMA) and vimentin, compared with fibroblasts (56).
CAFs produce numerous types of grow th factors, cytokines, chemokines, MMPs and ECM proteins that promote the progression of tumours. CAFs are able to stimulate angiogenesis via the secretion of VEGF, promote tumour cell proliferation via CXCL12, induce tumour cell invasion via TGF- $\beta 1$ and decrease tumour cell death via an anti-apoptosis factor, secreted frizzled-related protein 1 (55).

As for the sources of CAFs, a previous study that reported the origin of myofibroblasts in kidney fibrosis could provide implications (57). The results suggested that myofibroblasts arise from four sources, proliferation of resident fibroblasts (50\%), differentiation from bone marrow (35\%), and transition from endothelial (10\%) and epithelial (5\%) forms (57). However, the sources of CAFs in peritoneal metastasis nodes from gastric cancer have yet to be fully elucidated. Fuyuhiro et al (58) reported that the ratios of myofibroblasts to fibroblasts in tumour tissue are significantly higher compared with those in normal gastric tissue. Additionally, the ability of gastric cancer cells to migrate and invade is increased by CAFs, a function that may be attenuated using Smad2 siRNA and an anti-TGF- $\beta$ antibody (58). These results indicated that CAFs have a crucial role in the growth and invasion of gastric cancer cells (58). Another previous study verified that HPMCs are one source of CAFs (25). HPMCs have a spindle-shaped morphology following the stimulation of TGF- $\beta$ and these activated HPMCs are more invasive. The molecular mechanisms by which PMCs are incorporated into tumour structures have also been clarified (59). A previous study demonstrated that PMCs create the invasion front of peritoneal metastasis in a manner dependent on the activation of a tyrosine kinase substrate with five SH3 domains, a substrate of Src kinase (59). Furthermore, it has recently been identified that CAFs originate from bone marrow-derived cells (60). Bone marrow-derived cells that adopt a fibroblast-like morphology in the circulation are referred to as fibrocytes. Fibrocytes express the cell markers of leucocytes (CD45) as well as the markers of mesenchymal cells (collagen I). In addition, elevated levels of CXCL12 in gastric cancer cells and of CXCR4 in fibrocytes can be detected, indicating that CXCL12/CXCR4 may participate in the migration of fibrocytes from the bone marrow to gastric cancer sites (60).

Taken together, CAFs in gastric cancer originate from orthotopic fibroblasts, HPMCs and bone marrow-derived cells, and they promote the progression of gastric cancer in a number of ways $(25,58,60)$. The CXCL12/CXCR4 axis may be required in the migration of CAFs. In the tumour microenvironment, TGF- $\beta 1$, which originates from cancer cells and CAFs, has an important role in the interaction between cancer cells and CAFs (61). TGF- $\beta 1$ secreted by cancer cells promotes the transition to myofibroblasts and, in turn, myofibroblasts can secrete TGF- $\beta 1$, which further stimulates the proliferation of cancer cells (61).

An increasing number of studies have focused on the underlying mechanisms by which CAFs promote the progression of gastric cancer. The CAFs in gastric cancer have high expression levels of HGF and are one of the sources of secreted HGF in the microenvironment (62). HGF enhances the proliferation and migration of gastric cancer cells via paracrine signalling (62). A number of ECM proteins secreted from CAFs also have a role in the promotion of gastric cancer, 
including asporin and periostin $(63,64)$. Asporin belongs to a family of small leucine-rich proteoglycans and is a primary non-collagen component of ECM (65). Asporin is typically secreted by CAFs into the microenvironment of gastric cancer (63). Asporin in the microenvironment promotes invasion of CAFs and surrounding cancer cells through the CD44/Rac1 pathway (63). Periostin (POSTN), produced by fibroblasts, participates in the adhesion and migration of epithelial cells (66). Kikuchi et al (64) reported that POSTN is secreted by CAFs and enhances the proliferation of gastric cancer cells via the activation of ERK. In vivo investigation has further demonstrated that co-inoculation of gastric cancer cells with fibroblasts expressing POSTN facilitates tumour formation (64). The ECM proteins secreted by CAFs provide an optimal microenvironment for gastric cancer. Furthermore, a multitude of molecules, including growth, transcription and inflammatory factors, are also highly expressed in CAFs and stimulate the invasion of gastric cancer cells; these include fibroblast growth factor 9 (67), Twist 1 (68), interleukin 22 (69), galectin-1 (70) and transgelin (71). Consequently, CAFs, as important components of stromal cells, secrete numerous molecules that further promote tumourigenesis.

\section{Milky spots}

Another metastatic process of peritoneal dissemination is translymphatic metastasis. Lymphatic orifices, also known as stomata, are the gates on the peritoneal surface to the underside of capillary lymphatic vessels (72). Furthermore, there are gaps amongst the mesothelial cells covering stomata; therefore, peritoneal-free cancer cells are able to invade the submesothelial space through these stomata (73). The lack of the physical barrier presented by the compact HPMC monolayer indicates that translymphatic metastasis does not require numerous stages, unlike transmesothelial metastasis. Therefore translymphatic metastasis is frequently developed at an earlier stage compared with transmesothelial metastasis (73). Stomata are primarily located in the greater omentum, diaphragm, small bowel mesentery, pelvic peritoneum and falciform ligament, whilst the anterior abdominal wall, liver capsule, surface of the gastric wall and small bowel do not typically have lymphatic orifices (7). Hagiwara et al (73) initially identified milky spots on the peritoneum and malignant cells were revealed to implant into these sites. Milky spots are located in the greater omentum, mesenterium and pelvic floor in humans (7). However, there are no milky spots on the anterior parietal peritoneum, liver capsule or serosal surface of the small bowel (73). Therefore, these peritoneal regions are not invaded until the late stages of peritoneal metastasis with massive malignant ascites (73). Milky spots are specific to lymphoid tissue on the peritoneum and consist primarily of macrophages, as well as a number of lymphocytes and mastocytes (74). There are numerous lymphatic orifices on the surface of milky spots and macrophages migrate into the peritoneal cavity via these stomata (74).

As a lymphatic apparatus, milky spots have a vital role in peritoneal defence (75). However, it appears contradictory that a number of previous studies have reported that peritoneal-free cancer cells preferentially localize on milky spots $(73,76)$. There are numerous reasons for this paradox, besides the stomata exposing the submesothelial tissue to the cancer cells. Firstly, the levels of cellular adhesion molecules, including intercellular adhesion molecule 1, of the mesothelial cells that are on the surface of milky spots are increased compared with in other peritoneal regions, which may promote cell adhesion (77). Secondly, Oosterling et al (76) observed that the milky spots are insufficient to prevent peritoneal dissemination even in minimal residual disease, suggesting that an omentectomy may be required to prevent peritoneal dissemination for certain intra-abdominal malignancies. Thirdly, when exposed to gastric cancer cells, peritoneal milky spot macrophages remodel into an alternative active macrophage (M2 macrophages) phenotype, which are designated as tumour-associated macrophages (TAMs) (78). TAMs have been established to promote angiogenesis and the proliferation of cancer cells (79). A previous study demonstrated that, when co-cultured with gastric cancer cells, macrophages have downregulated the expression of CD80, CD86 and major histocompatibility complex-II, but also upregulated the expression of CD206 (78). These TAMs further induce HPMC apoptosis and fibroblastic transformation, providing an optimal microenvironment for the growth of gastric cancer cells.

Recently, the C-C motif chemokine ligand 22 (CCL22)/CC chemokine receptor 4 (CCR4) axis has been the focus of more studies as it participates in the infiltration of gastric cancer cells into milky spots. A previous study established that gastric cancer cells express CCR4, and that CCL22 may also be detected within omental milky spots (80). CCL22 increases the proliferation and migration of gastric cancer cells in vitro (80). These results indicate that CCL22/CCR4 may participate in this selective infiltration process (80). Furthermore, milky spots are rich in capillaries and the surrounding vascular density is high (81). CD105+ vessels and vascular sprouts have been also located within milky spots, indicating that angiogenesis is active (81). Additionally, milky spots also serves as a hypoxic niche, which enhances the self-renewing capacity of gastric cancer stem/progenitor cells regulated by hypoxia-inducible factor- $1 \alpha(\mathrm{HIF}-1 \alpha)(82)$.

Consequently, milky spots, as a lymphatic apparatus, participate in the immune defence of the peritoneal cavity. However, stomata on the milky spots provide a gate via which peritoneal-free cancer cells may migrate into the submesothelial space.

\section{Involvement of microRNA in peritoneal dissemination}

Microarray analysis has been used to systematically search aberrantly expressed microRNA (miRNA) that is associated with human cancer. miRNAs are small non-coding RNAs that have a role in the post-transcriptional regulation of gene expression. An increasing number of studies have demonstrated that miRNAs are associated with tumour development and progression $(83,84)$. Certain miRNAs have been established to be associated with peritoneal dissemination in gastric cancer (85-87). Zheng et al (85) reported that microRNA (miR)-409-3p inhibited the ability of gastric cancer cells to invade and metastasise via targeting radixin. Another previous study demonstrated that miR-495 and miR-551a suppressed gastric cancer cell migration and invasion by targeting PRL-3, which exhibits significantly increased expression levels in 
primary gastric cancer cells with peritoneal dissemination (86). miR-25 was identified to enhance gastric cancer progression by targeting Erb-B2 receptor tyrosine kinase 2 both in vitro and in vivo (87). Other miRNAs have been reported to correlate with peritoneal dissemination in gastric cancer, including miR-200b (88), miR-125a-3q (89) and let-7 miRNAs (90). These studies, regarding miRNAs, present a novel therapeutic target for suppressing peritoneal dissemination in gastric cancer. Takei et al (91) established an orthotopic implantation model for the peritoneal metastasis of gastric cancer and demonstrated that inoculation with mi-516a-3q cells resulted in significantly increased survival periods, compared with inoculation with control cells.

\section{Transvessel metastasis theory}

Paget's 'seed and soil' theory is the mainstream theory for the formation of peritoneal dissemination in gastric cancer. However, a number of studies have questioned the validity of this theory and have proposed certain alternative mechanisms to explain the progress of peritoneal dissemination. Miyake et al (92) suggested a hypothesis wherein peritoneal dissemination is a type of transvessel metastasis mediated by HIF-1 $\alpha$. HIF- $1 \alpha$ is a subunit of the heterodimeric transcription factor HIF-1 and is an important regulator of the cellular response to hypoxia. Recent studies have implied that hypoxia participates in each step of the cancer metastasis cascade, including EMT, invasion, intravasation, homing and proliferation to secondary organs (93-95). All of these results have indicated that HIF-1 $\alpha$ may be a vital regulator in cancer metastasis. Miyake et al (92) established orthotopic implantation (o.i.) and conventional intraperitoneal injection (i.p.) models to mimic the peritoneal dissemination of gastric cancer. Results showed that HIF-1 $\alpha$ was crucial for the development of peritoneal dissemination in the o.i. model, whereas it had an inhibitory role in the peritoneal dissemination in the i.p. model (92). These results confirm the angiogenesis function of HIF-1 $\alpha$ and indicate the 'seeding' theory may not be correct (92). In addition, another previous study has suggested that the subperitoneal space comprises blood vessels, the lymphatics, lymph nodes and fatty tissue, which provide an important passageway for cancer cells within the peritoneal cavity (96). This previous study supports the transvessel metastasis hypothesis to a certain extent. However, further studies are required to validate the accuracy of this theory.

\section{Conclusion}

A number of previous studies have focused on the molecules involved in the peritoneal dissemination of gastric cancer, and each step of the process demonstrates the stimulation of various molecules, including growth factors, cytokines and chemokines. Furthermore, microarray analysis has been increasingly used to systematically identify metastasis-associated genes, including their associated mRNAs and miRNAs. Additionally, it may be possible to predict, prevent and treat peritoneal metastasis by targeting these molecules. Previous studies have primarily focused on the characteristics of malignant cancer cells, but an increasing number of studies have focused on the tumour microenvironment, particularly milky spots and the tumour stroma $(54,64)$. Milky spots are specific sites for peritoneal metastasis and numerous studies have demonstrated that milky spots are the first metastasis location for free cancer cells. Stroma, as a component of tumour tissue, interacts with cancer cells and promotes cell invasion and migration. However, the most frequently used peritoneal dissemination model was established using the i.p. model of gastric cancer cells. This model does not include the initial steps of dissemination, such as invasion in the gastric wall and the detachment from the primary tumour. Therefore, a more persuasive orthotopic implantation model would mimic the formation of peritoneal dissemination. The underlying mechanisms of peritoneal metastasis remain in dispute. The transvessel metastasis theory has been recently proposed by Miyake et al (92) and provides more information and the novel therapeutic target HIF-1 $\alpha$. However, further investigation is required in order to validate this hypothesis.

\section{Acknowledgements}

The present study was supported by the National Natural Science Foundation of China (grant no. 81372364).

\section{References}

1. Stewart BW and Wild CP: World cancer report 2014. World, 2014.

2. Japanese Gastric Cancer Association Registration Committee; Maruyama K, Kaminishi M, Hayashi K, Isobe Y, Honda I, Katai H, Arai K, Kodera Y and Nashimoto A: Gastric cancer treated in 1991 in Japan: Data analysis of nationwide registry. Gastric Cancer 9: 51-66, 2006.

3. Maehara Y, Hasuda S, Koga T, Tokunaga E, Kakeji Y and Sugimachi K: Postoperative outcome and sites of recurrence in patients following curative resection of gastric cancer. $\mathrm{Br}$ J Surg 87: 353-357, 2000.

4. Yashiro M, Chung YS, Nishimura S, Inoue T and Sowa M: Fibrosis in the peritoneum induced by scirrhous gastric cancer cells may act as 'soil' for peritoneal dissemination. Cancer 77 (8 Suppl): S1668-S1675, 1996.

5. Li Z, Miao Z, Jin G, Li X, Li H, Lv Z and Xu HM: big-h3 supports gastric cancer cell adhesion, migration and proliferation in peritoneal carcinomatosis. Mol Med Rep 6: 558-564, 2012.

6 . Paget $\mathrm{S}$ : The distribution of secondary growths in cancer of the breast. Cancer Metastasis Rev 8: 98-101, 1989.

7. Yonemura Y, Kawamura T, Bandou E, Tsukiyama G, Endou Y and Miura M: The natural history of free cancer cells in the peritoneal cavity. Recent Results Cancer Res 169: 11-23, 2007.

8. Bando E, Yonemura Y, Takeshita Y, Taniguchi K, Yasui T, Yoshimitsu Y, Fushida S, Fujimura T, Nishimura G and Miwa K: Intraoperative lavage for cytological examination in 1,297 patients with gastric carcinoma. Am J Surg 178: 256-262, 1999.

9. Yonemura Y, Endou Y, Kimura K, Fushida S, Bandou E, Taniguchi K, Kinoshita K, Ninomiya I, Sugiyama K, Heizmann CW, et al: Inverse expression of S100A4 and E-cadherin is associated with metastatic potential in gastric cancer. Clin Cancer Res 6: 4234-4242, 2000.

10. Marutsuka T, Shimada S, Shiomori K, Hayashi N, Yagi Y, Yamane $\mathrm{T}$ and Ogawa M: Mechanisms of peritoneal metastasis after operation for non-serosa-invasive gastric carcinoma: An ultrarapid detection system for intraperitoneal free cancer cells and a prophylactic strategy for peritoneal metastasis. Clin Cancer Res 9: 678-685, 2003.

11. Takebayashi K, Murata S, Yamamoto H, Ishida M, Yamaguchi T, Kojima M, Shimizu T, Shiomi H, Sonoda H, Naka S, et al: Surgery-induced peritoneal cancer cells in patients who have undergone curative gastrectomy for gastric cancer. Ann Surg Oncol 21: 1991-1997, 2014.

12. Zlotnik A: Chemokines and cancer. Int J Cancer 119: 2026-2029, 2006. 
13. Tanaka T, Bai Z, Srinoulprasert Y, Yang BG, Hayasaka H and Miyasaka M: Chemokines in tumor progression and metastasis. Cancer Sci 96: 317-322, 2005.

14. Yoshie O: Immune chemokines and their receptors: The key elements in the genesis, homeostasis and function of the immune system. Springer Semin Immunopathol 22: 371-391, 2000.

15. Yasumoto K, Koizumi K, Kawashima A, Saitoh Y, Arita Y, Shinohara K, Minami T, Nakayama T, Sakurai H, Takahashi Y, et al: Role of the CXCL12/CXCR4 axis in peritoneal carcinomatosis of gastric cancer. Cancer Res 66: 2181-2187, 2006.

16. Müller A, Homey B, Soto H, Ge N, Catron D, Buchanan ME, McClanahan T, Murphy E, Yuan W, Wagner SN, et al: Involvement of chemokine receptors in breast cancer metastasis. Nature 410: $50-56,2001$.

17. Koizumi K, Hojo S, Akashi T, Yasumoto K and Saiki I: Chemokine receptors in cancer metastasis and cancer cell-derived chemokines in host immune response. Cancer Sci 98: 1652-1658, 2007.

18. Lv ZD, Na D, Liu FN, Du ZM, Sun Z, Li Z, Ma XY, Wang ZN and $\mathrm{Xu} H \mathrm{HM}$ : Induction of gastric cancer cell adhesion through transforming growth factor-beta1-mediated peritoneal fibrosis. J Exp Clin Cancer Res 29: 139, 2010.

19. Kiyasu Y, Kaneshima S and Koga S: Morphogenesis of peritoneal metastasis in human gastric cancer. Cancer Res 41: 1236-1239, 1981.

20. Lamouille S, Xu J and Derynck R: Molecular mechanisms of epithelial-mesenchymal transition. Nat Rev Mol Cell Biol 15: 178-196, 2014

21. Thiery JP, Acloque H, Huang RY and Nieto MA: Epithelialmesenchymal transitions in development and disease. Cell 139: 871-890, 2009

22. Margetts $\mathrm{PJ}, \mathrm{Oh} \mathrm{KH}$ and Kolb $\mathrm{M}$ : Transforming growth factor-beta: Importance in long-term peritoneal membrane changes. Perit Dial Int 25 (Suppl 3): S15-S17, 2005.

23. Massagué J: TGF $\beta$ signalling in context. Nat Rev Mol Cell Biol 13: 616-630, 2012

24. Gordon KJ and Blobe GC: Role of transforming growth factor-beta superfamily signaling pathways in human disease Biochim Biophys Acta 1782: 197-228, 2008.

25. Tsukada T, Fushida S, Harada S, Yagi Y, Kinoshita J, Oyama K, Tajima H, Fujita H, Ninomiya I, Fujimura T and Ohta T: The role of human peritoneal mesothelial cells in the fibrosis and progression of gastric cancer. Int J Oncol 41: 476-482, 2012.

26. Liu Q, Mao H, Nie J, Chen W, Yang Q, Dong X and Yu X: Transforming growth factor \{beta\}1 induces epithelial-mesenchymal transition by activating the JNK-Smad3 pathway in rat peritoneal mesothelial cells. Perit Dial Int 28 (Suppl 3): S88-S95, 2008.

27. Lv ZD, Wang HB, Li FN, Wu L, Liu C, Nie G, Kong B, Qu HL and Li JG: TGF- $\beta 1$ induces peritoneal fibrosis by activating the Smad2 pathway in mesothelial cells and promotes peritoneal carcinomatosis. Int J Mol Med 29: 373-379, 2012.

28. Jiang CG, Lv L, Liu FR, Wang ZN, Na D, Li F, Li JB, Sun Z and $\mathrm{Xu} \mathrm{HM}$ : Connective tissue growth factor is a positive regulator of epithelial-mesenchymal transition and promotes the adhesion with gastric cancer cells in human peritoneal mesothelial cells. Cytokine 61: 173-180, 2013.

29. Okazaki M, Fushida S, Harada S, Tsukada T, Kinoshita J, Oyama K, Tajima H, Ninomiya I, Fujimura T and Ohta T: The angiotensin II type 1 receptor blocker candesartan suppresses proliferation and fibrosis in gastric cancer. Cancer Lett 355: 46-53, 2014

30. Shinbo T, Fushida S, Tsukada T, Harada S, Kinoshita J, Oyama K, Okamoto K, Ninomiya I, Takamura $\mathrm{H}$, Kitagawa $\mathrm{H}$, et al: Protein-bound polysaccharide $\mathrm{K}$ suppresses tumor fibrosis in gastric cancer by inhibiting the TGF-b signaling pathway. Oncol Rep 33: 553-558, 2015.

31. Tanaka H, Muguruma K, Ohira M, Kubo N, Yamashita Y, Maeda K, Sawada T and Hirakawa K: Impact of adjuvant immunochemotherapy using protein-bound polysaccharide-K on overall survival of patients with gastric cancer. Anticancer Res 32: 3427-3433, 2012.

32. Ono Y, Hayashida T, Konagai A, Okazaki H, Miyao K, Kawachi S, Tanabe M, Shinoda M, Jinno H, Hasegawa H, et al: Direct inhibition of the transforming growth factor- $\beta$ pathway by protein-bound polysaccharide through inactivation of Smad2 signaling. Cancer Sci 103: 317-324, 2012

33. Nakashio T, Narita T, Akiyama S, Kasai Y, Fujiwara M, Ito K, Takagi $\mathrm{H}$ and Kanngi R: Adhesion of human gastric and pancreatic cancer cells to peritoneal mesothelial cells is mediated by CD44 and beta(1) integrin. Int J Oncol 10: 183-188, 1997.
34. Takatsuki H, Komatsu S, Sano R, Takada Y and Tsuji T: Adhesion of gastric carcinoma cells to peritoneum mediated by alpha3beta1 integrin (VLA-3). Cancer Res 64: 6065-6070, 2004.

35. Giancotti FG and Ruoslahti E: Integrin signaling. Science 285: 1028-1032, 1999.

36. Fukuda K, Saikawa Y, Yagi H, Wada N, Takahashi T and Kitagawa Y: Role of integrin a1 subunits in gastric cancer patients with peritoneal dissemination. Mol Med Rep 5: 336-340, 2012.

37. Nishimura S, Chung YS, Yashiro M, Inoue T and Sowa M: Role of alpha 2 beta 1- and alpha 3 beta 1-integrin in the peritoneal implantation of scirrhous gastric carcinoma. Br J Cancer 74: 1406-1412, 1996.

38. Chen CN, Chang CC, Lai HS, Jeng YM, Chen CI, Chang KJ, Lee $\mathrm{PH}$ and Lee $\mathrm{H}$ : Connective tissue growth factor inhibits gastric cancer peritoneal metastasis by blocking integrin a3b1-dependent adhesion. Gastric Cancer 18: 504-515, 2015.

39. Yonemura Y, Endo Y, Fujita H, Kimura K, Sugiyama K, Momiyama N, Shimada $\mathrm{H}$ and Sasaki T: Inhibition of peritoneal dissemination in human gastric cancer by MMP-7-specific antisense oligonucleotide. J Exp Clin Cancer Res 20: 205-212, 2001

40. Li Z, Zhang D, Zhang H, Miao Z, Tang Y, Sun G and Dai D: Prediction of peritoneal recurrence by the mRNA level of CEA and MMP-7 in peritoneal lavage of gastric cancer patients. Tumour Biol 35: 3463-3470, 2014.

41. Mimori K, Fukagawa T, Kosaka Y, Ishikawa K, Iwatsuki M, Yokobori T, Hirasaki S, Takatsuno Y, Sakashita H, Ishii H, et al: A large-scale study of MT1-MMP as a marker for isolated tumor cells in peripheral blood and bone marrow in gastric cancer cases. Ann Surg Oncol 15: 2934-2942, 2008.

42. Visse R and Nagase H: Matrix metalloproteinases and tissue inhibitors of metalloproteinases: Structure, function, and biochemistry. Circ Res 92: 827-839, 2003.

43. Miyagi M, Aoyagi K, Kato S and Shirouzu K: The TIMP-1 gene transferred through adenovirus mediation shows a suppressive effect on peritoneal metastases from gastric cancer. Int J Clin Oncol 12: 17-24, 2007.

44. Yoshikawa T, Tsuburaya A, Kobayashi O, Sairenji M, Motohashi H, Yanoma S and Noguchi Y: Intratumoral concentrations of tissue inhibitor of matrix metalloproteinase 1 in patients with gastric carcinoma a new biomartker for invasion and its impact on survival. Cancer 91: 1739-1744, 2001.

45. Gentile A, Trusolino L and Comoglio PM: The met tyrosine kinase receptor in development and cancer. Cancer Metastasis Rev 27: 85-94, 2008

46. Trusolino L, Bertotti A and Comoglio PM: MET signalling: Principles and functions in development, organ regeneration and cancer. Nat Rev Mol Cell Biol 11: 834-848, 2010.

47. Toiyama Y, Yasuda H, Saigusa S, Matushita K, Fujikawa H, Tanaka K, Mohri Y, Inoue Y, Goel A and Kusunoki M: Co-expression of hepatocyte growth factor and c-Met predicts peritoneal dissemination established by autocrine hepatocyte growth factor/c-Met signaling in gastric cancer. Int J Cancer 130: 2912-2921, 2012

48. Binder DK and Scharfman HE: Brain-derived neurotrophic factor. Growth Factors 22: 123-131, 2004

49. Okugawa Y, Tanaka K, Inoue Y, Kawamura M, Kawamoto A, Hiro J, Saigusa S, Toiyama Y, Ohi M, Uchida K, et al: Brain-derived neurotrophic factor/tropomyosin-related kinase B pathway in gastric cancer. Br J Cancer 108: 121-130, 2013.

50. Perretti M and D'Acquisto F: Annexin A1 and glucocorticoids as effectors of the resolution of inflammation. Nat Rev Immunol 9: 62-70, 2009.

51. Leoni G, Alam A, Neumann PA, Lambeth JD, Cheng G, McCoy J, Hilgarth RS, Kundu K, Murthy N, Kusters D, et al: Annexin A1, formyl peptide receptor, and NOX1 orchestrate epithelial repair. J Clin Invest 123: 443-454, 2013.

52. Cheng TY, Wu MS, Lin JT, Lin MT, Shun CT, Huang HY, Hua KT and Kuo ML: Annexin A1 is associated with gastric cancer survival and promotes gastric cancer cell invasiveness through the formyl peptide receptor/extracellular signal-regulated kinase/integrin beta-1-binding protein 1 pathway. Cancer 118: 5757-5767, 2012.

53. Aoyagi K, Kouhuji K, Yano S, Miyagi M, Imaizumi T, Takeda J and Shirouzu K: VEGF significance in peritoneal recurrence from gastric cancer. Gastric Cancer 8: 155-163, 2005.

54. Mueller MM and Fusenig NE: Friends or foes-bipolar effects of the tumour stroma in cancer. Nat Rev Cancer 4: 839-849, 2004.

55. Shimoda M, Mellody KT and Orimo A: Carcinoma-associated fibroblasts are a rate-limiting determinant for tumour progression. Semin Cell Dev Biol 21: 19-25, 2010. 
56. Gabbiani G, Ryan GB and Majne G: Presence of modified fibroblasts in granulation tissue and their possible role in wound contraction. Experientia 27: 549-550, 1971.

57. LeBleu VS, Taduri G, O'Connell J, Teng Y, Cooke VG, Woda C, Sugimoto $\mathrm{H}$ and Kalluri R: Origin and function of myofibroblasts in kidney fibrosis. Nat Med 19: 1047-1053, 2013.

58. Fuyuhiro Y, Yashiro M, Noda S, Matsuoka J, Hasegawa T, Kato Y, Sawada T and Hirakawa K: Cancer-associated orthotopic myofibroblasts stimulates the motility of gastric carcinoma cells. Cancer Sci 103: 797-805, 2012.

59. Satoyoshi R, Aiba N, Yanagihara K, Yashiro M and Tanaka M: Tks5 activation in mesothelial cells creates invasion front of peritoneal carcinomatosis. Oncogene 34: 3176-3187, 2015.

60. Terai S, Fushida S, Tsukada T, Kinoshita J, Oyama K, Okamoto K, Makino I, Tajima H, Ninomiya I, Fujimura T, et al: Bone marrow derived 'fibrocytes' contribute to tumor proliferation and fibrosis in gastric cancer. Gastric Cancer 18: 306-313, 2015.

61. Ostman A and Augsten M: Cancer-associated fibroblasts and tumor growth-bystanders turning into key players. Curr Opin Genet Dev 19: 67-73, 2009

62. Wu X, Chen X, Zhou Q, Li P, Yu B, Li J, Qu Y, Yan J, Yu Y, Yan M, et al: Hepatocyte growth factor activates tumor stromal fibroblasts to promote tumorigenesis in gastric cancer. Cancer Lett 335: 128-135, 2013

63. Satoyoshi R, Kuriyama S, Aiba N, Yashiro M and Tanaka M: Asporin activates coordinated invasion of scirrhous gastric cancer and cancer-associated fibroblasts. Oncogene 34: 650-660, 2015.

64. Kikuchi Y, Kunita A, Iwata C, Komura D, Nishiyama T, Shimazu K, Takeshita K, Shibahara J, Kii I, Morishita Y, et al: The niche component periostin is produced by cancer-associated fibroblasts, supporting growth of gastric cancer through ERK activation. Am J Pathol 184: 859-870, 2014

65. Nakajima M, Kizawa H, Saitoh M, Kou I, Miyazono K and Ikegawa S: Mechanisms for asporin function and regulation in articular cartilage. J Biol Chem 282: 32185-32192, 2007.

66. Liu Y and Liu BA: Enhanced proliferation, invasion, and epithelial-mesenchymal transition of nicotine-promoted gastric cancer by periostin. World J Gastroenterol 17: 2674-2680, 2011.

67. Sun C, Fukui H, Hara K, Zhang X, Kitayama Y, Eda H, Tomita T, Oshima T, Kikuchi S, Watari J, et al: FGF9 from cancer-associated fibroblasts is a possible mediator of invasion and anti-apoptosis of gastric cancer cells. BMC Cancer 15: 333, 2015.

68. Sung CO, Lee KW, Han S and Kim SH: Twist1 is up-regulated in gastric cancer-associated fibroblasts with poor clinical outcomes. Am J Pathol 179: 1827-1838, 2011.

69. Fukui H, Zhang X, Sun C, Hara K, Kikuchi S, Yamasaki T, Kondo T, Tomita T, Oshima T, Watari J, et al: IL-22 produced by cancer-associated fibroblasts promotes gastric cancer cell invasion via STAT3 and ERK signaling. Br J Cancer 111: 763-771, 2014.

70. He XJ, Tao HQ, Hu ZM, Ma YY, Xu J, Wang HJ, Xia YJ, Li L, Fei BY, Li YQ and Chen JZ: Expression of galectin-1 in carcinoma-associated fibroblasts promotes gastric cancer cell invasion through upregulation of integrin b1. Cancer Sci 105: $1402-1410,2014$

71. Yu B, Chen X, Li J, Qu Y, Su L, Peng Y, Huang J, Yan J, Yu Y, $\mathrm{Gu}$ Q, et al: Stromal fibroblasts in the microenvironment of gastric carcinomas promote tumor metastasis via upregulating TAGLN expression. BMC Cell Biol 14: 17, 2013.

72. Shimotsuma M, Shields JW, Simpson-Morgan MW, Sakuyama A, Shirasu M, Hagiwara A and Takahashi T: Morpho-physiological function and role of omental milky spots as omentum-associated lymphoid tissue (OALT) in the peritoneal cavity. Lymphology 26 90-101, 1993.

73. Hagiwara A, Takahashi T, Sawai K, Taniguchi H, Shimotsuma M, Okano S, Sakakura C, Tsujimoto H, Osaki K, Sasaki S, et al: Milky spots as the implantation site for malignant cells in peritoneal dissemination in mice. Cancer Res 53: 687-692, 1993.

74. Beelen RH, Fluitsma DM and Hoefsmit EC: The cellular composition of omentum milky spots and the ultrastructure of milky spot macrophages and reticulum cells. J Reticuloendothel Soc 28: 585-599, 1980.

75. Liebermann-Meffert D: The greater omentum. Anatomy, embryology, and surgical applications. Surg Clin North Am 80: 275-293, xii, 2000

76. Oosterling SJ, van der Bij GJ, Bögels M, van der Sijp JR, Beelen RH, Meijer S and van Egmond M: Insufficient ability of omental milky spots to prevent peritoneal tumor outgrowth supports omentectomy in minimal residual disease. Cancer Immunol Immunother 55: 1043-1051, 2006.
77. Cui L, Johkura K, Liang Y, Teng R, Ogiwara N, Okouchi Y, Asanuma K and Sasaki K: Biodefense function of omental milky spots through cell adhesion molecules and leukocyte proliferation. Cell Tissue Res 310: 321-330, 2002.

78. Liu XY, Miao ZF, Zhao TT, Wang ZN, Xu YY, Gao J, Wu JH, You Y, Xu H and Xu HM: Milky spot macrophages remodeled by gastric cancer cells promote peritoneal mesothelial cell injury. Biochem Biophys Res Commun 439: 378-383, 2013.

79. Bingle L, Brown NJ and Lewis CE: The role of tumour-associated macrophages in tumour progression: Implications for new anticancer therapies. J Pathol 196: 254-265, 2002.

80. Cao L, Hu X, Zhang J, Huang G and Zhang Y: The role of the CCL22-CCR4 axis in the metastasis of gastric cancer cells into omental milky spots. J Transl Med 12: 267, 2014.

81. Gerber SA, Rybalko VY, Bigelow CE, Lugade AA, Foster TH, Frelinger JG and Lord EM: Preferential attachment of peritoneal tumor metastases to omental immune aggregates and possible role of a unique vascular microenvironment in metastatic survival and growth. Am J Pathol 169: 1739-1752, 2006.

82. Miao ZF, Wang ZN, Zhao TT, Xu YY, Gao J, Miao F and Xu HM: Peritoneal milky spots serve as a hypoxic niche and favor gastric cancer stem/progenitor cell peritoneal dissemination through hypoxia-inducible factor 1a. Stem Cells 32: 3062-3074, 2014.

83. Esquela-Kerscher A and Slack FJ: Oncomirs - microRNAs with a role in cancer. Nat Rev Cancer 6: 259-269, 2006.

84. Zhang B, Pan X, Cobb GP and Anderson TA: microRNAs as oncogenes and tumor suppressors. Dev Biol 302: 1-12, 2007.

85. Zheng B, Liang L, Huang S, Zha R, Liu L, Jia D, Tian Q, Wang Q, Wang C, Long Z, et al: MicroRNA-409 suppresses tumour cell invasion and metastasis by directly targeting radixin in gastric cancers. Oncogene 31: 4509-4516, 2012.

86. Li Z, Cao Y, Jie Z, Liu Y, Li Y, Li J, Zhu G, Liu Z, Tu Y, Peng G, et al: miR-495 and miR-551a inhibit the migration and invasion of human gastric cancer cells by directly interacting with PRL-3. Cancer Lett 323: 41-47, 2012.

87. Li BS, Zuo QF, Zhao YL, Xiao B, Zhuang Y, Mao XH, Wu C, Yang SM, Zeng H, Zou QM and Guo G: MicroRNA-25 promotes gastric cancer migration, invasion and proliferation by directly targeting transducer of ERBB2, 1 and correlates with poor survival. Oncogene 34: 2556-2565, 2015.

88. Kurashige J, Mima K, Sawada G, Takahashi Y, Eguchi H, Sugimachi K, Mori M, Yanagihara K, Yashiro M, Hirakawa K, et al: Epigenetic modulation and repression of miR-200b by cancer-associated fibroblasts contribute to cancer invasion and peritoneal dissemination in gastric cancer. Carcinogenesis 36 : 133-141, 2015.

89. Hashiguchi Y, Nishida N, Mimori K, Sudo T, Tanaka F, Shibata K, Ishii H, Mochizuki H, Hase K, Doki Y and Mori M: Down-regulation of miR-125a-3p in human gastric cancer and its clinicopathological significance. Int J Oncol 40: 1477-1482, 2012

90. Ohshima K, Inoue K, Fujiwara A, Hatakeyama K, Kanto K, Watanabe Y, Muramatsu K, Fukuda Y, Ogura S, Yamaguchi K and Mochizuki T: Let-7 microRNA family is selectively secreted into the extracellular environment via exosomes in a metastatic gastric cancer cell line. PLoS One 5: e13247, 2010.

91. Takei Y, Takigahira M, Mihara K, Tarumi Y and Yanagihara K: The metastasis-associated microRNA miR-516a-3p is a novel therapeutic target for inhibiting peritoneal dissemination of human scirrhous gastric cancer. Cancer Res 71: 1442-1453, 2011.

92. Miyake S, Kitajima Y, Nakamura J, Kai K, Yanagihara K, Tanaka T, Hiraki M, Miyazaki K and Noshiro H: HIF-la is a crucial factor in the development of peritoneal dissemination via natural metastatic routes in scirrhous gastric cancer. Int J Oncol 43: 1431-1440, 2013.

93. Krishnamachary B,Zagzag D, Nagasawa H, Rainey K, OkuyamaH, Baek JH and Semenza GL: Hypoxia-inducible factor-1dependent repression of E-cadherin in von Hippel-Lindau tumor suppressor-null renal cell carcinoma mediated by TCF3, ZFHX1A, and ZFHX1B. Cancer Res 66: 2725-2731, 2006.

94. Funasaka T and Raz A: The role of autocrine motility factor in tumor and tumor microenvironment. Cancer Metastasis Rev 26: 725-735, 2007.

95. Lu X and Kang Y: Hypoxia and hypoxia-inducible factors: Master regulators of metastasis. Clin Cancer Res 16: 5928-5935, 2010.

96. Silverman PM: The subperitoneal space: Mechanisms of tumour spread in the peritoneal cavity, mesentery, and omentum. Cancer Imaging 4: 25-29, 2003 